\title{
Grupos de investigación colaborativa: ventajas e impacto en la investigación quirúrgica, un Ilamado desde ColombianSurg
}

\section{Collaborative research groups: advantages and impact in surgical research, a call from ColombianSurg}

\author{
María Alejandra Pineda ${ }^{1} \mathbb{D}$, Laura C. Arce-Polania ${ }^{1} \mathbb{D}$, Lilian Torregrosa-Almonacid² $\mathbb{D}$, \\ Luis Felipe Cabrera-Vargas ${ }^{3}$, Carlos J. Pérez-Rivera ${ }^{4} \mathbb{D}$, en nombre de ColombianSurg
}

1 Médica, grupo de investigación en Cirugía General, Fundación Cardioinfantil - Instituto de Cardiología, Bogotá, D.C., Colombia.

2 MD, FACS, especialista en Cirugía general, Cirugía de Seno y Tejidos Blandos y magíster en Bioética; profesora asociada de Cirugía, Directora Departamento de Cirugía y Especialidades, Facultad de Medicina, Pontificia Universidad Javeriana, Hospital Universitario San Ignacio. Presidente, Asociación Colombiana de Cirugía, Bogotá, D.C., Colombia.

3 Médico, especialista en Cirugía General, Fundación Santa Fe de Bogotá; profesor de cirugía, Universidad de los Andes y Universidad el Bosque; coordinador del capítulo Futuros Cirujanos, Asociación Colombiana de Cirugía, Bogotá, D.C., Colombia.

4 Médico, magister en Epidemiología, coordinador del grupo de investigación en Cirugía General, Fundación Cardioinfantil - Instituto de Cardiología, Bogotá, D.C., Colombia.

La medicina basada en la evidencia surge como una estrategia para superar la práctica médica apoyada en la racionalidad fisiopatológica y la experiencia clínica no sistematizada ${ }^{1}$. A lo largo de su desarrollo, ha generado la necesidad de adoptar una mayor responsabilidad frente a la investigación, como un aspecto importante del crecimiento profesional médico. Actualmente, se considera una aptitud clave en el desempeño de la medicina, asociada a la capacidad de contribuir a la investigación clínica, en beneficio de la atención del paciente. De hecho, las competencias investigativas, de comprensión y análisis crítico, de las publicaciones médico-científicas, son fundamentales para una buena práctica clínica ${ }^{2,3}$.

Palabras clave: cirugía general; academias e institutos; grupos de investigación; política de investigación en salud; ética en investigación; evaluación de la investigación en salud.

Keywords: general surgery; academies and institutes; research groups; health research policy; ethics, research; health research evaluation.

Fecha de recibido: 29/01/2021 - Fecha de aceptación: 04/02/2021 - Fecha de publicación en línea: 29/03/2021

Correspondencia: Carlos J. Pérez-Rivera, Calle 163A \# 13B - 60, Departamento de Investigaciones, Fundación Cardioinfantil - Instituto de Cardiología, Bogotá, D.C., Colombia. Teléfono: 6672727 ext. 73203. correo electrónico: cjperezrivera@gmail.com

Citar como: Pineda MA, Arce-Polania LC, Torregrosa-Almonacid L, Cabrera-Vargas LF, Pérez-Rivera CJ. Grupos de investigación colaborativa: ventajas e impacto en la investigación quirúrgica, un Ilamado desde ColombianSurg. Rev Colomb Cir. 2021;36:391-6. https://doi.org/10.30944/20117582.883

Este es un artículo de acceso abierto bajo una Licencia Creative Commons - BY-NC-ND https://creativecommons.org/licenses/by-ncnd/4.0/deed.es 


\section{¿Cuáles son las limitaciones en la realización de proyectos de investigación?}

La literatura es unánime en cuanto a la importancia de adquirir experiencia y fortalecimiento en competencias investigativas de primera mano ${ }^{2}$. No obstante, los proyectos de investigación realizados durante procesos educativos de pregrado, posgrado e incluso, en la práctica profesional, generalmente son llevados a cabo de forma independiente, abarcando a menudo una población de estudio reducida. Esto se refleja en una limitación para el desarrollo de la investigación quirúrgica, resultando en proyectos realizados en un solo centro de atención de salud, que incluyen un bajo número de pacientes y que, finalmente, generan datos institucionales de bajo impacto en la toma de decisiones médicas, de modo que el tiempo requerido para planificar, gestionar y ejecutar proyectos de investigación de alta calidad representa una gran dificultad ${ }^{2,3}$.

\section{¿Qué es un grupo de investigación colaborativa?}

Los conceptos y características acerca de los grupos de investigación colaborativos que se plantean en la actualidad (tabla 1), describen organizaciones conformadas por redes de profesionales de la salud y otras áreas, dispuestos a realizar investigación multicéntrica ${ }^{2}$. Una definición, concreta y universal, aún no es clara y no se cuenta con una estandarización de dichos conceptos; sin embargo, es posible plantear una definición a partir de las experiencias registradas.

Se consideran entonces como redes de personas interesadas en la investigación científica, que trabajan en equipo, para llevar a cabo proyectos investigativos con objetivos comunes. Son grupos multidisciplinarios, que no incluyen de forma exclusiva personal de la salud o en formación, sino también profesionales de otras áreas, que comparten el mismo interés de investigación quirúrgica (tabla 2).

La investigación colaborativa es llevada a cabo por grupos de personas encargadas de ejecutar estudios relevantes y potencialmente realizables, con alta calidad científica, que generan un efecto en la toma de decisiones de la práctica médica diaria, ya sea de forma individual o generando información fundamental para influir en las estadísticas nacionales y globales. Todo lo anterior, con el propósito de optimizar la atención de los pacientes y permitir la protección y promoción de la salud en el paciente quirúrgico ${ }^{2,3}$.

Tabla 1. Definiciones de la literatura acerca de los grupos de investigación colaborativa.

\begin{tabular}{|l|l|}
\hline Definición & Fuente \\
\hline $\begin{array}{l}\text { "En el nivel más simple, las colaboraciones de investigación son redes de personas } \\
\text { interesadas que reconocen los beneficios de trabajar juntos para realizar una } \\
\text { investigación. Actúan juntos como un mecanismo de filtrado para identificar estudios } \\
\text { que probablemente sean atractivos, relevantes y entregables. También actúan como } \\
\text { una "agencia de citas", comparando a los aprendices con otros aprendices con intereses } \\
\text { similares que han adquirido una mayor experiencia de investigación y poniendo a } \\
\text { of } \\
\text { ambos gollaborative. }{ }^{2}\end{array}$ & \\
$\begin{array}{l}\text { "Grupo de persons en contacto con apoyo académico." } \\
\text { proyectos de investigación, que trabajan con un enfoque dirigido a la meta-investigación } \\
\text { como la síntesis de evidencia y la evaluación crítica de la calidad metodológica y de } \\
\text { informes de la investigación." }\end{array}$ & \\
\hline $\begin{array}{l}\text { Definimos una investigación colaborativa de aprendices quirúrgicos como una } \\
\text { organización o grupo, dirigido principalmente por aprendices o estudiantes de medicina, } \\
\text { que realiza investigación quirúrgica multicéntrica basada en pacientes. }\end{array}$ & $\begin{array}{l}\text { Jamjoom AAB, Phan PNH, } \\
\text { Hutchinson PJ, Kolias AG. }{ }^{5}\end{array}$ \\
\hline $\begin{array}{l}\text { "Grupo de investigación centrado en investigar y mejorar las metodologías de } \\
\text { investigación, la realización de la investigación y el cumplimiento de informes dentro } \\
\text { de la investigación quirúrgica, utilizando metainvestigación, equipos pequeños con alta } \\
\text { autonomía individual y la entrega de resultados de investigación rápidos." }\end{array}$ & $\begin{array}{l}\text { Pidgeon TE, Chandrakumar C, Al } \\
\text { Omran Y, Limb C, Thavayogan R, }\end{array}$ \\
\hline
\end{tabular}


Tabla 2. Ventajas y desventajas de los grupos de investigación colaborativa

\begin{tabular}{|c|c|}
\hline Ventajas & Desventajas $^{3}$ \\
\hline $\begin{array}{l}\text { - Puede participar cualquier estudiante o profesional interesado } \\
\text { en realizar investigación. } \\
\text { - Los nombres de todos los contribuyentes son citables en } \\
\text { PudMed (sujeto a los criterios de la revista). } \\
\text { - La inversión económica para realizar estudios multicéntricos, } \\
\text { según la modalidad de investigación colaborativa, es menor } \\
\text { en comparación con la metodología tradicional. } \\
\text { - Pretende mejorar la calidad de atención de los pacientes, } \\
\text { optimizando la toma de decisiones médicas a nivel poblacional. } \\
\text { - Las colaboraciones de investigación producen mayor número } \\
\text { de publicaciones de alta calidad, con gran impacto en la } \\
\text { práctica médica y en menor tiempo. } \\
\text { - Optimiza los resultados en los proyectos investigativos, al } \\
\text { estudiar diferentes tipos de poblaciones. }\end{array}$ & $\begin{array}{l}\text { - La nomenclatura desarrollada en la actualidad para autorías } \\
\text { colaborativas carece de validación formal. } \\
\text { - Aunque la estructura de la autoría colaborativa refleja la } \\
\text { utilizada por grandes ensayos clínicos multicéntricos, no } \\
\text { es ampliamente aceptada como evidencia de actividad } \\
\text { investigadora por parte de los organismos de formación en } \\
\text { cirugía a lo largo de la ruta de formación de pre y posgrado, } \\
\text { seguramente por la novedad que representa en nuestro } \\
\text { país. }\end{array}$ \\
\hline
\end{tabular}

\section{¿Cómo funcionan los grupos de investigación colaborativa?}

El trabajo científico adopta una metodología dirigida a la colaboración por medio de la conformación de redes, tanto de talento humano como de instituciones de salud. La investigación colaborativa pretende, por medio del uso de la tecnología de la información y la comunicación, generar una dispersión geográfica de investigadores que aborden intereses comunes, al dar acceso a datos y herramientas que permitan obtener procesos de investigación de alta calidad. Implica que los grupos dedicados a realizar este tipo de investigación desarrollen destrezas comunicativas y prácticas de trabajo en equipo, compartidas en razón del mismo objetivo.

Actualmente, no existe un consenso o guía que describa este tipo de investigación colaborativa, sin embargo, existen registros acerca del éxito y los objetivos que se han logrado con el desarrollo de dicha metodología. Se especifican contribuciones y recomendaciones acerca de la conformación de estos grupos, teniendo en cuenta la práctica y la experiencia en diferentes partes del mundo ${ }^{4-6}$.

En la revisión de literatura, se evidencian algunas publicaciones que incentivan su implementación, junto con una descripción de las competencias que deben hacer parte del perfil de cada integrante del grupo de investigación, según su función, responsabilidad en el proyecto y comité al que pertenece, dentro de los que se resalta la capacidad de entender los principios básicos de la dirección en investigación y cómo deben aplicarse las directrices éticas pertinentes propias de las actividades investigativas ${ }^{3}$. No obstante, dada la estructura institucional de salud en Colombia, surge la necesidad de implementar nuevos comités para optimizar el desempeño de un grupo de investigación colaborativa, los cuales se detallan en la tabla 3.

De acuerdo con lo planteado anteriormente, teniendo en cuenta el éxito con el que GlobalSurg, EuroSurg, ItalianSurg y otros, han desarrollado numerosos proyectos de investigación colaborativa, se hace necesaria la implementación de este tipo de grupo de investigación en el país, como pretende ser ColombianSurg, cuyo objetivo general es consolidar una comunidad multidisciplinaria, de personas pertenecientes al gremio de la salud como cirujanos generales y subespecialistas, médicos generales, epidemiólogos y salubristas, y estudiantes de medicina, además de estadísticos, ingenieros, diseñadores y todo aquel interesado en el área quirúrgica y el cuidado perioperatorio, con el fin de crear y compartir conocimiento que impulse el desarrollo profesional en el ámbito investigativo, realizando proyectos que impacten en el desarrollo de las políticas públicas en nuestro país. 
Tabla 3. Roles y responsabilidades en los grupos de investigación colaborativa.

\begin{tabular}{|c|c|}
\hline Rol & Responsabilidad \\
\hline Comité directivo & $\begin{array}{l}\text { - Involucrado en la organización general del proyecto. } \\
\text { - Instrumental en la concepción, desarrollo y administración del proyecto. } \\
\text { - Diseña y administra las herramientas de recolección de datos } \\
\text { - Supervisa el plan de difusión de los resultados del proyecto. }\end{array}$ \\
\hline Comité académico & $\begin{array}{l}\text { - Dirige los estudios investigativos } \\
\text { - Verifica la información aportada } \\
\text { - Proporciona una revisión crítica periódica del plan y el protocolo del estudio. } \\
\text { - Revisión del manuscrito previo a su publicación }\end{array}$ \\
\hline Comité de planeación & $\begin{array}{l}\text { - Comunicación con los diferentes líderes sobre los proyectos que serán llevados a cabo. } \\
\text { - Creación del protocolo (junto con comité de escritura) } \\
\text { - Desarrollo de documentación necesaria para presentar el proyecto ante los comités de } \\
\text { investigación y de ética } \\
\text { - Coordinación de la vinculación de las instituciones interesadas a través de correos y posterior } \\
\text { - inscripción en la plataforma RedCap. } \\
\text { - Verificación del cumplimiento de las fechas propuestas } \\
\text { - Envío del manuscrito final a la revista, junto con su debida documentación. }\end{array}$ \\
\hline Comité de redacción & $\begin{array}{l}\text { - Revisa la base de evidencia existente relevante para este manuscrito. } \\
\text { - Contribución significativa del trabajo original a una o más secciones del manuscrito } \\
\text { - Búsqueda de potenciales revistas para enviar la publicación y redacción de respectivos correos } \\
\text { - Revisa y edita críticamente el manuscrito. } \\
\text { - Realiza correcciones sugeridas por la revista }\end{array}$ \\
\hline Comité de comunicación & $\begin{array}{l}\text { - Creación de contenido para redes sociales } \\
\text { - Crea infografía y logo del estudio por cada proyecto que se cree o vincule }\end{array}$ \\
\hline $\begin{array}{l}\text { Comité de metodología y } \\
\text { análisis }\end{array}$ & $\begin{array}{l}\text { - Formula el plan de análisis estadístico } \\
\text { - Utiliza los datos producidos en un estudio para resumir resultados, realizar pruebas } \\
\text { estadísticas y generar conclusiones. } \\
\text { - Realiza auditorias para la evaluación de la calidad de los datos. }\end{array}$ \\
\hline $\begin{array}{l}\text { Líder institucional } \\
\text { (Jefe del departamento } \\
\text { de cirugía de cada } \\
\text { institución) }\end{array}$ & $\begin{array}{l}\text { - Responsable de difundir el proyecto y los centros de reclutamiento dentro de su institución } \\
\text { - Responsable de presentar el estudio en reuniones regionales de educación e investigación. } \\
\text { - Asegura que todos los consultores relevantes dentro de la institución estén al tanto del estudio. } \\
\text { - Actúa de enlace con el investigador principal para organizar el registro local y la aprobación } \\
\text { del estudio. }\end{array}$ \\
\hline $\begin{array}{l}\text { Investigador principal } \\
\text { institucional }\end{array}$ & $\begin{array}{l}\text { - Lidera el proyecto dentro de una sola institución (puede ser un solo hospital o una } \\
\text { organización compuesta por varios hospitales). } \\
\text { - Recluta y gestiona colaboradores locales. } \\
\text { - Presenta estudio en reuniones departamentales y hospitalarias locales. }\end{array}$ \\
\hline Equipo investigador & $\begin{array}{l}\text { - Realiza la recolección de los datos. } \\
\text { - Revisa los borradores del manuscrito. } \\
\text { - Participa en la evaluación de la calidad de los datos. }\end{array}$ \\
\hline
\end{tabular}

Así mismo, se establece como visión de ColombianSurg, potenciar el progreso científico de las especialidades quirúrgicas en el país, convirtiéndose en un líder y referente nacional en la investigación quirúrgica y el cuidado perioperatorio, en conjunto con las distintas instituciones que deseen construir evidencia científica, permitiendo la formulación y desarrollo de estudios de alta calidad. Creemos que de esta manera se logrará potenciar el progreso científico de las especialida- des que tienen un rol en el cuidado perioperatorio del paciente, impactando positivamente en la salud pública, a nivel nacional y global, como meta a mediano y a largo plazo.

Para ello se creó un proyecto que pretende incentivar la vinculación de cualquier colombiano interesado en la investigación quirúrgica colaborativa, inscribiéndose a través del enlace https://forms.gle/L2MEU3jzmb3TrGEM9, lo que permitirá la adjunción y el desarrollo de esta inno- 
vadora propuesta en el país, la cual pretende ser un pilar para el desarrollo de estudios multicéntricos dentro del territorio nacional y promover la colaboración como metodología de investigación científica sobresaliente.

Este editorial tiene como finalidad impulsar la investigación colaborativa y la creación de lazos entre las distintas instituciones de salud del país, cirujanos, estudiantes y profesionales de diferentes áreas, mediante la realización de estudios multicéntricos de alta calidad, por medio de ColombianSurg como grupo de investigación colaborativa, pionero en desarrollar este tipo de metodología científica a nivel nacional, y que actualmente, ya que cuenta con los instrumentos necesarios para desarrollar este tipo de proyectos, garantiza un rápido flujo para el intercambio de información.

Agradecimientos: Expresamos nuestros sinceros agradecimientos a todo el equipo de ColombianSurg (Apéndice 1) por el trabajo realizado para el desarrollo de la investigación quirúrgica del país.

\section{Cumplimiento de normas éticas}

Consentimiento informado: Por tratarse de una revisión de la literatura, este es un estudio sin riesgo de acuerdo con lo definido en la resolución de 008430 de 1993 del Ministerio de Salud de Colombia, por lo que no se requirió consentimiento informado ni aprobación del Comité de Ética Institucional.

Conflictos de intereses: No se declaró ningún conflicto de interés por parte de los autores.

Financiación: autofinanciado por los autores.

\section{Contribución de los autores:}

Concepción y diseño del estudio: María Alejandra Pineda, Laura C. Arce-Polania, Lilian Torregrosa, Luis Felipe Cabrera, Carlos J. Pérez-Rivera.

Redacción del manuscrito: María Alejandra Pineda, Laura C. Arce-Polania.
Revisión crítica: Lilian Torregrosa, Luis Felipe Cabrera, Carlos J. Pérez-Rivera.

Aprobación final del manuscrito: María Alejandra Pineda, Laura C. Arce-Polania, Lilian Torregrosa, Luis Felipe Cabrera, Carlos J. Pérez-Rivera.

\section{Referencias}

1. Málaga G, Neira-Sánchez ER. La medicina basada en la evidencia, su evolución a 25 años desde su diseminación, promoviendo una práctica clínica científica, cuidadosa, afectuosa y humana. Acta Med Peru. 2018;35:121-6.

2. Bartlett D, Pinkney TD, Futaba K, Whisker L, Dowswell G, et al, on behalf of the West Midlands Research Collaborative. Trainee led research collaboratives: pioneers in the new research landscape. BMJ. 2012; 345:e5084. https://doi.org/10.1136/bmj.e5084

3. Blencowe N, Glasbey J, Heywood N, Kasivisvanathan V, Lee M, Nepogodiev D, et al, on behalf of The National Research Collaborative \& Association of Surgeons in Training Collaborative Consensus Group. Recognising contributions to work in research collaboratives: Guidelines for standardising reporting of authorship in collaborative research. Int J Surg. 2018;52:355-60. https://doi.org/10.1016/j.ijsu.2017.12.019

4. The Academic Surgical Collaborative, Pidgeon TE, Fowler AJ, Whitehurst K, Wellstead G, Gundogan B, et al. The Academic Surgical Collaborative: Launching a new trainee research collaborative. Ann Med Surg. 2015;4:133-5.

https://doi.org/10.1016/j.amsu.2015.04.020

5. Jamjoom AAB, Phan PNH, Hutchinson PJ, Kolias AG. Surgical trainee research collaboratives in the UK: An observational study of research activity and publication productivity. BMJ open. 2016;6(2):e010374. https://doi.org/10.1136/bmjopen-2015-010374

6. Nepogodiev D, Chapman SJ, Kolias AG, Fitzgerald JE, Lee M, Blencowe NS, on behalf of the National Surgical Research Collaborative. The effect of trainee research collaboratives in the UK. Lancet Gastroenterol Hepatol. 2017;2:247-8. https://doi.org/10.1016/S2468-1253(17)30033-X

7. Pidgeon TE, Chandrakumar C, Omran YA, Limb C, Thavayogan R, Gundogan B, et al. The Academic Surgical Collaborative: A three-year review of a trainee research collaborative. Annals of Medicine and Surgery. 2018;28:38-44. https://doi.org/10.1016/j.amsu.2018.01.003 


\section{Apéndice 1}

\section{ColombianSurg}

\begin{tabular}{|l|l|}
\hline COMITÉ ACADÉMICO & COMITÉ DIRECTIVO \\
\hline Lilian Torregrosa-Almonacid & Carlos José Pérez-Rivera \\
\hline Luis Felipe Cabrera-Vargas & Juan Guillermo Barrera-Carvajal \\
\hline Leonardo Briceño-Ayala & Paulo Andrés Cabrera-Rivera \\
\hline COMITÉ DE PLANEACIÓN & COMITÉ DE REDACCIÓN \\
\hline Lina M. Acosta-Buitrago & Sandra Contreras-Arrieta \\
\hline Laura Cristina Arce-Polania & Alejandro González-Orozco \\
\hline María Alejandra Pineda-Castañeda & Camilo Andrés Polania-Sandoval \\
\hline Valeria Cormane-Alfaro & Isabella Velandia-Sánchez \\
\hline Juan David Saavedra & Juan Pablo García-Méndez \\
\hline & Julián Alberto Garavito-González \\
\hline & Valeria Patricia Bustos-Hemer \\
\hline COMITÉ DE COMUNICACIÓN & COMITÉ DE METODOLOGÍA Y ANÁLISIS \\
\hline Ruth Eliana Pineda-Mateus & Camilo Alejandro Velandia-Sánchez \\
\hline María Paz Bohórquez-Tarazona & Michelle Ruíz-Pérez \\
\hline Julián Miguel Corso-Ramírez & Nicolás Lozano-Suárez \\
\hline Andrea Barragán-Méndez & María Paula Vargas-Cuellar \\
\hline & Danna Lesley Cruz-Reyes \\
\hline & Carlos Felipe Durán-Torres \\
\hline & Daniel Alejandro Buitrago-Medina \\
\hline & Ana María Barragán-González \\
\hline & \\
\hline
\end{tabular}

